\title{
Spina bifida: A few simple facts about a complex condition
}

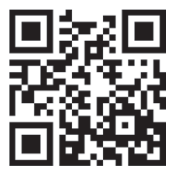

Open spina bifida (also known as myelomeningocele $(\mathrm{SBM}))$ is the most complex birth defect involving the nervous system, and children born with this condition face a lifetime of medical care with numerous interventions. It is hard to think of another condition where initial management has such a profound effect on survival and ability.

The March edition of CME is on SBM - in fact, there are so many dimensions to this condition that the editor of CME has chosen to spread this topic over two editions of the journal.

There are a few good reasons why SBM remains of relevance to health professionals in the 21st century, especially in South Africa. Firstly, SBM is often preventable. There is overwhelming evidence that women administered folic acid from before conception up to 12 weeks into pregnancy, either as a vitamin supplement or through food fortification, have a substantial reduction in their risk of having a baby with a neural tube defect, such as SBM. Although the risk is reduced by around two-thirds in most series, it is not abolished, pointing to a multifactorial aetiology, with other environmental and as yet unidentified genetic factors playing a role.

A programme of folic acid fortification of staple foods was implemented in South Africa a decade ago. Although this has had an impact, the quantity may not be sufficient, and taking a supplement is advisable, especially in high-risk pregnancies. All healthcare workers in South Africa must be aware of these facts and never miss an opportunity to educate prospective parents. Simply stated, any woman at risk of pregnancy should take folic acid supplements.

Secondly, SBM may be diagnosed early enough in pregnancy for prospective parents to be counselled about options such as continuation or termination of the pregnancy. As this is always a very difficult decision, counselling must be non-directive, ensuring that the parents are well informed and then fully supported in their decision, either way. Although screening should be considered in all pregnancies, this may not be possible throughout South Africa. However, women with high-risk pregnancies must be referred.

With modern ultrasound technology and expertise, in most cases there is simply no excuse for missing the diagnosis.

For babies born with SBM, the key to management is awareness and early diagnosis of the more common problems facing them.
This includes immediate resuscitation and referral for closure of the open lesion within 24 hours (which must surely be achievable in South Africa), timely treatment of hydrocephalus, early urological management to prevent renal damage, and early developmental assessment to ensure appropriate educational support. While the majority of patients have normal intelligence, there is great variability in the needs and outcomes of this group.

Long-term management includes the expertise of a wide range of other professionals, including orthopaedic surgeons (with the help of orthotists), stomatherapists, physiotherapists, occupational therapists, psychologists, and social workers. The role of the radiologist must not be overlooked, especially with regard to radiation exposure - given the enormous number of investigations that may be required.

Care is best offered in the context of a multidisciplinary clinic, but regrettably this is seldom the case and management is often fragmented, both in private practice and the state sector. A primary care doctor who ensures holistic care is of great value. With increasing long-term survival there has been greater appreciation of the challenges faced by adolescents with neurodisablity, and more attention is now paid to transition of care into adulthood.

Given the complexity of this condition, it is hardly surprising that it has stimulated numerous ethical debates with implications that go beyond the spina bifida population, as exemplified by Lorber's selection criteria in the UK, the Baby Jane Doe case in the USA, and the Groningen protocol for euthanasia of newborns in the Netherlands. These approaches may be contrasted to the recent enthusiasm for fetal surgery, which in turn has generated further dilemmas.

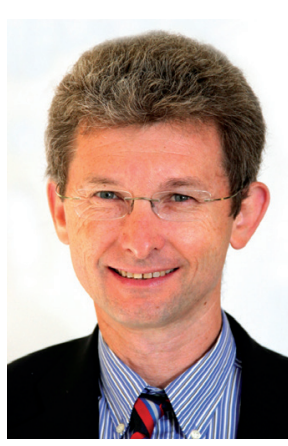

When all is said and done, managing these patients well is very gratifying. Despite the many problems they face, hope and support enable a rewarding and meaningful life.

\section{Graham Fieggen \\ Guest editor \\ graham.fieggen@uct.ac.za}

S Afr Med J 2014;104(3):212. DOI:10.7196/SAMJ.8094 